\title{
6 Zum Einfluss des Deutschen auf das Tschechische: Die Effekte des Zeitdrucks auf die Sprachproduktion
}

\subsection{Einführung}

Es ist zweifellos so, dass die deutsche und die tschechische Kultur, und somit auch die beiden Sprachen, jahrhundertelang aufeinander gewirkt und sich gegenseitig beeinflusst haben (vgl. Berger 2003 - Lautsystem; Berger 2008 Übersichtsartikel; Schmiedtová 2010 - Temporalität; Behrens et al. 2014 - Syntax). Es stellt sich jedoch die Frage, wie tief dieser Einfluss greift bzw. für welche sprachlichen Bereiche der Einfluss des Deutschen im Tschechischen aufgezeigt werden kann (siehe Kap. 3, 4 und 10).

Sehr gut dokumentiert ist der Einfluss im Bereich des Lexikons (vgl. Havránek/Fischer (Hgg.) 1965; Schmiedtová/Schmiedtová 1996). So konnte zum Beispiel in der Studie von Schmiedtová/Schmiedtová (1996) zur Anwendung von lexikalischen Germanismen im modernen Tschechisch anhand von korpusbelegtem ${ }^{95}$ Sprachmaterial nachgewiesen werden, dass Germanismen einen festen Status im Lexikon des Tschechischen haben und dass sie von tschechischen Muttersprachlern produktiv gebraucht werden.

Aus weiteren, teilweise älteren Arbeiten geht hervor, dass der Einfluss des deutschen auf das tschechische System nicht auf den lexikalischen Bereich beschränkt bleibt: Es zeigt sich, dass verbale Präfixe im Deutschen und Tschechischen überraschend viele Gemeinsamkeiten aufweisen (Schmiedtová 2003a, 2003b). Aber auch in Bezug auf Partikeln (Nekula 1996), Demonstrativpronomina (Berger 1993; Borthen et al. 2012), Flexion (Havránek 1937; Rösler 1952) und Syntax (Hausenblas 1958; Behrens et al. 2014) lassen sich in den zwei aus typologischer Sicht unterschiedlichen Sprachen bestimmte Ähnlichkeiten feststellen.

Die in diesem Kapitel präsentierte Studie untersucht den Einfluss des Deutschen auf das grammatische und konzeptuelle System des Tschechischen. ${ }^{96}$ Die Grundlage dafür stellt insbesondere das Kapitel 5 dar, in dem gezeigt wurde, dass unter gewöhnlichen Zeitbedingungen Sprecher verschiedener Muttersprachen

95 Die Korpora, die für diese Studie verwendet wurden, sind alle Teil des Tschechischen Nationalkorpus (vgl. auch: http://ucnk.ff.cuni.cz).

96 Um die Ähnlichkeiten zwischen den Systemen beider Sprachen besser aufzeigen und diese auf den Sprachkontakt mit dem Deutschen zurückführen zu können, wird zusätzlich das Russische - als eine zweite slawische Sprache - zum Vergleich herangezogen.

Ә Open Access. (C) 2018 Mertins, publiziert von De Gruyter. (œ) [r-NC-ND Dieses Werk ist lizenziert unter der Creative Commons Attribution-NonCommercial-NoDerivatives 4.0 Lizenz. 
zielorientierte Bewegungsereignisse unterschiedlich konzeptualisieren, verbalisieren und im Gedächtnis speichern und von dort abrufen. Nun stellt sich die Frage, welche Bestandteile des präsentierten Ereignisses für die Konzeptualisierung/Verbalisierung obligatorisch sind. Dies wird mit Hilfe des ZeitdruckParadigmas in einem Elizitationsexperiment untersucht (siehe auch Kap. 1, Abschn. 1.3). Der diesem experimentellen Paradigma zugrundeliegende Gedanke besteht darin, eine Situation zu kreieren, in der die Probanden aufgrund der stark begrenzten Zeit für die Planung die Redeinhalte auf die absolut notwendigen Informationen reduzieren. Mit Hilfe dieses Paradigmas lassen sich Informationen, die für die Konzeptualisierung und Versprachlichung von Ereignissen nicht auslassbar bzw. obligatorisch sind, elizitieren. Es ist dabei anzunehmen, dass bei der Selektion von Informationen neben den für die Planung der Sprachproduktion zugrundeliegenden Prinzipien auch sprachstrukturelle Merkmale eine wichtige Rolle spielen.

Die hier vorgestellte Studie knüpft an eine Reihe kürzlich erschienener Arbeiten zum Vergleich von Sprachproduktionsdaten im Deutschen und Tschechischen an (vgl. Schmiedtová/von Stutterheim/Carroll 2011; Schmiedtová 2011a, 2011b, 2012; Kap. 3 und 4), in denen neben linguistischen Daten auch Blickbewegungs-, Sprechanfangszeit- und Gedächtnisdaten analysiert wurden. Diese Studien belegen, dass der Einfluss des Deutschen sich auch auf sprachliche Bereiche wie Informationsstruktur, Informationsselektion und Perspektivierung erstreckt. Die Tatsache, dass beispielsweise in der Zuteilung der visuellen Aufmerksamkeit (Blickbewegungsverhalten) tschechische und deutsche Muttersprachler sehr ähnliche Muster zeigen, weist darauf hin, dass sich aus sprachtypologischer Sicht das Deutsche und das Tschechische deutlich näherstehen, als bisher angenommen wurde.

\subsection{Theoretischer Hintergrund}

Der theoretische Ansatz von Sapir/Whorf (vgl. Whorf 1956) sowie die Thinking for Speaking-Hypothese (Slobin 1996a) stellen den Ausgangspunkt für die in diesem Kapitel vorgestellte Studie dar (siehe auch Kap. 5). Es wird angenommen, dass bestimmte sprachliche Merkmale einer Sprache eine entscheidende Auswirkung auf die Wahrnehmung der Außenwelt und so auf deren sprachliche Beschreibung haben (siehe auch Kap. 1, Abschn. 1.1).

Darüber hinaus nimmt dieses Kapitel wesentlich Bezug auf eine Reihe neuerer Arbeiten, die zeigen, wie unterschiedlich Sprecher verschiedener Sprachen Ereignisse konzeptualisieren und versprachlichen und welche Rolle dabei sprachstrukturelle Merkmale spielen (vgl. Carroll/Lambert 2003; von Stutter- 
heim/Nüse 2003). Es wird dabei angenommen, dass solche Merkmale in gewisser Weise als gefrorene Konzeptualisierungsschemata funktionieren. Diese Konzeptualisierungsschemata sind sprachspezifisch und beeinflussen die sprachlichen Präferenzen, über die Sprecher in ihrer Muttersprache verfügen. Um welche sprachstrukturellen Merkmale handelt es sich hier konkret? Es geht um die grammatische Kategorie des Aspekts, eine verbale Kategorie, die obligatorisch am Verb markiert wird (siehe Kap. 1, Abschn. 1.1; Kap. 2)

In früheren Studien (vgl. Carroll/von Stutterheim/Nüse 2004) wurde erstmals gezeigt, dass sich Sprachen im Hinblick auf die Enkodierung von Ereignissen erheblich unterscheiden und diese Unterschiede damit korrelieren, ob eine Sprache über eine grammatische Aspektkategorie verfügt oder nicht. Dies spiegelt sich in den unterschiedlichen Perspektiven wider, welche Sprecher systematisch einnehmen, wenn sie ein- und dasselbe Ereignis versprachlichen (siehe Kap. 5 und 7). In der einschlägigen Literatur werden zwei Perspektiven unterschieden, die Sprecher verwenden, wenn sie zielorientierte Bewegungsereignisse konzeptualisieren und verbalisieren: Die im Deutschen bevorzugte Perspektive ist die holistische Perspektive, die für englischsprachige Sprecher typische Perspektive die Verlaufsperspektive.

Solche sprachspezifischen Konzeptualisierungsschemata spiegeln sich nicht nur in sprachlichen (vgl. Carroll/Lambert 2003) und nicht-sprachlichen Daten (Kap. 5), sondern auch in Sprechanfangszeiten (Schmiedtová 2011a) und Blickbewegungsmessungen wider (Kap. 5).

Generell gilt, dass der Einfluss der sprachlichen Strukturen auf die Konzeptualisierung sich nicht ausschließlich aufgrund von kontrastiven sprachlichen Daten begründen lässt, da eine solche Argumentation tautologisch wäre (= Wenn zwischensprachliche Unterschiede vorhanden sind, müssen auch konzeptuelle Unterschiede bestehen). Um dieses Problem auszuschließen, müssen zusätzlich Daten aus nicht-sprachlichen Bereichen (z.B. Gedächtnis-, Sortierungsaufgaben etc.) und/oder chronometrischen Messungen (z.B. Blickbewegungen, ereigniskorrelierte Potentiale) herangezogen werden (vgl. Lucy 1996 - ein Artikel mit wichtigen methodologischen Überlegungen und Hinweisen zur Erforschung der Beziehung von Sprache und Kognition).

\subsubsection{Eigene Vorarbeiten: Endpunkte}

Für dieses Kapitel sind von der Autorin mehrere Studien einschlägig, deren Fokus auf der Enkodierung von Bewegungsereignissen im Deutschen, Tschechischen und Russischen liegt (vgl. Schmiedtová/von Stutterheim/Carroll 2011; Schmiedtová 2011a; Kap. 5 und 8). Im Folgenden wird auf diese Studien näher eingegan- 
gen. In der in Schmiedtová/von Stutterheim/Carroll (2011) und Kapitel 8 vorgestellten Studie wird gezeigt, dass sich tschechische Muttersprachler von Muttersprachlern des Russischen hinsichtlich der Endpunktmarkierung unterscheiden: Muttersprachler des Tschechischen erwähnen bedeutend mehr Endpunkte für die Darstellung der kritischen Szenen, als dies russische Sprecher tun. Tschechische Muttersprachler gehen also so vor, dass sie Endpunkte in allen kritischen Szenen in der Mehrheit der Äußerungen verbalisieren. Die russischen Muttersprachler dagegen scheinen in diesem Bereich eine andere Vorgehensweise zu haben: Sie erwähnen bevorzugt erst dann die Endpunkte, wenn diese in einem Clip auch tatsächlich als erreicht gezeigt werden.

Deutsche Muttersprachler erwähnen in allen kritischen Szenen mehr Endpunkte als russische Muttersprachler. Dabei ist die Präferenz der Endpunktmarkierung der deutschen und tschechischen Muttersprachler vergleichbar: Für Szenen, in denen der Endpunkt als erreicht dargestellt wird [+END], wurden für die L1-Daten keine statistisch relevanten Unterschiede festgestellt. Die Ergebnisse der linguistischen Analysen wurden in einer weiteren Studie zur Endpunktmarkierung in sieben verschiedenen Sprachen bestätigt (Kap. 5). Auch diese Studie hat belegt, dass deutsche und tschechische Sprecher Ereignisse systematisch unter der holistischen Perspektive wiedergeben, während russische Sprecher für den gleichen Ereignistyp - die Verlaufsperspektive verwenden. Auch die Ergebnisse des Gedächtnistests (Kap. 5) haben deutlich gemacht, dass sich deutsche und tschechische Sprecher in Bezug auf die kritischen Stimuli besser an die Endpunkte erinnern als russische Sprecher. Bei den Kontrollszenen wiederum wurden keine statistisch relevanten Unterschiede festgestellt, was zeigt, dass die für die kritischen Stimuli herausgefundenen zwischensprachlichen Unterschiede nicht durch individuell unterschiedliche Gedächtnisleistungen erklärt werden können.

Die Ergebnisse der Sprach- und Gedächtnistests werden durch die Befunde aus der Messung der Sprechanfangszeiten weiter gestützt (Schmiedtová 2011a). Für kritische [-END]-Szenen hat sich ergeben, dass bei deutschen und tschechischen Muttersprachlern die Sprechanfangszeiten gegenüber den russischen Muttersprachlern signifikant verzögert sind. Die Versprachlichung von Bewegungsereignissen des Typs [-END] beginnt also deutlich später. In den Kontrollszenen konnte kein Unterschied festgestellt werden.

\subsubsection{Eigene Vorarbeiten: Aspektgebrauch im Tschechischen und Russischen}

Für das Tschechische und das Russische ist gleichermaßen charakteristisch, dass sie mit zwei grammatischen Aspekten markieren, ob ein Ereignis zu Ende geht 
(= abgeschlossenes Ereignis - perfektiver Aspekt) oder sich im Verlauf befindet (= nicht abgeschlossenes Ereignis - imperfektiver Aspekt). Außerdem gibt es Aspektformen, die markieren können, ob sich ein Ereignis wiederholt oder habituell ist. Wie bereits in Kapitel 2 und 5 deutlich gemacht, verfügt das Deutsche über keine grammatische Aspektkategorie. Es stehen dennoch sprachliche Mittel zur Verfügung, die genutzt werden können, um ein Ereignis als abgeschlossen, im Verlauf oder als wiederholt zu umschreiben.

Entscheidend für die Konzeptualisierung bzw. die Planung einer Verbalisierung ist dabei, ob ein bestimmtes Konzept in der Sprache obligatorisch durch grammatische Mittel markiert wird (wie das Konzept 'nicht-abgeschlossen vs. abgeschlossen' im Russischen oder Tschechischen), oder ob ein solches Konzept wahlweise mit nicht-grammatischen Mitteln zum Ausdruck gebracht werden kann. Es gibt also eine Interdependenz zwischen den in der Grammatik einer Sprache vorhandenen sprachstrukturellen Merkmalen (z.B. Aspekt) und der Konzeptualisierung eines Ereignisses (z.B. 'nicht-abgeschlossen vs. abgeschlossen').

In der slawistischen Aspektforschung wurde bis heute kaum den Präferenzen Aufmerksamkeit gewidmet, die sich auf die tatsächliche Verwendung einer bestimmten morphologischen Verbalform beziehen. Das heißt, Aspektanalysen wurden kaum anhand von empirisch gestützten Daten durchgeführt. Die Ergebnisse von Schmiedtová/Sahonenko (2008) und Schmiedtová/von Stutterheim/ Carroll (2011) zeigen, dass Muttersprachler des Tschechischen und des Russischen trotz der Ähnlichkeiten in den zugrundeliegenden Aspektsystemen unterschiedliche Präferenzen für verschiedene morphologische Verbalformen in der Darstellung eines bestimmten Situationstyps zeigen.

Tschechische Muttersprachler verwenden präfigierte Verben, die vom grammatischen Aspekt her meistens perfektiv sind, sehr oft für Situationen, in denen es sich um den Nachzustand einer Handlung handelt. Eine charakteristische Versprachlichung einer Szene mit Nachzustand im Tschechischen ist: tsch. Paní vypila - (perfektive Vergangenheitsform) dt. 'Eine Frau trank ein Glas Wasser aus'. Die präfigierte Form lässt sich auch für Bewegungsereignisse vom Typ [-END] belegen: tsch. Pán ve-šel do domu (perfektive Vergangenheitsform) - dt. 'Ein Mann ging ins Haus rein'. In russischen Daten kommen diese Formen nur in jenen Szenen vor, in denen eine Bewegung von Punkt A zu Punkt B als erreicht gezeigt wird [+END] (siehe auch Kap. 3-4).

Sekundäre Imperfektiva, die vom grammatischen Aspekt her immer imperfektiv sind (siehe Kap. 8, Abschn. 8.2.1.2), werden von tschechischen Sprechern nur selten gebraucht. Dagegen kommen sie in russischen Daten sehr häufig vor und zwar vorwiegend in Szenen mit Ereignissen, die sich in mehrere Subintervalle einteilen lassen (z.B. die oben erwähnte Szene, in der eine Frau ein Glas Wasser austrinkt). 
Der markanteste Unterschied in der Anwendung der Aspektformen bezieht sich jedoch auf den Gebrauch der präfigierten perfektiven Präsensformen, die im Tschechischen auch für die Online-Darstellung der hier und jetzt ablaufenden Ereignisse gebraucht werden. In Bezug auf die o.g. Szene gebraucht die Versuchsperson die präfigierte perfektive Präsensform, während der Stimulus noch läuft (tsch. Paní vy-pije sklenici vody - dt. 'Eine Frau trinkt (perfektive Präsensform) ein Glas Wasser aus)'. Manchmal wird in diesem Kontext das temporale Adverb ted', právě ('jetzt') verwendet. Im Russischen dagegen werden präfigierte perfektive Präsensformen in Verbindung mit der Hier-und-jetzt-Bedeutung nie verwendet, da diese Kombination nicht grammatisch ist. Diese Aspektform hat im Russischen immer eine Zukunftsbedeutung. Die Unterschiede im Aspektgebrauch im Tschechischen und im Russischen werden durch die Studie von Dickey (2000) zu unterschiedlichen Grammatikalisierungen des Aspektes in den west- (Tschechisch) und ostslawischen Sprachen (Russisch) unterstützt. ${ }^{97}$

Zusammenfassend lässt sich festhalten, dass das Tschechische anders als das Russische die holistische Perspektive bei der Konzeptualisierung und Versprachlichung von zielorientierten Ereignissen verwendet und somit eine Gruppe mit dem Deutschen bildet. In Bezug auf den Aspektgebrauch in den beiden slawischen Sprachen können auch signifikante Unterschiede festgestellt werden: Im Gegensatz zum Russischen bevorzugt das Tschechische präfigierte perfektive Verbformen für die Versprachlichung von Ereignissen mit Nachzustand; in manchen Fällen kommt diese Form sogar in der Verbalisierung zielorientierter Bewegungsereignisse vor. Hervorzuheben ist dabei der Gebrauch der perfektiven Präsensformen in Verbindung mit der Hier-und-jetzt-Bedeutung, der im Tschechischen durchaus möglich, im Russischen jedoch ganz ausgeschlossen ist.

Die Befunde aus der Sprachproduktion werden durch die Ergebnisse einer Befragung von 256 tschechischen Muttersprachlern aus fünf verschiedenen Regionen Tschechiens bestätigt. Mit Hilfe eines Fragebogens wurden aspektuelle Präferenzen für eine Reihe von Hier-und-jetzt-Situationen überprüft. Die

97 Nach Dickey (2000) stellt das polnische aspektuelle System hinsichtlich des Grammatikalisierungsgrades einen Übergang zwischen der west- und ostslawischen Sprachgruppe dar. In Bezug auf Aspektgebrauch in zielorientierten Ereignissen sowie Ereignissen mit Nachzustand haben die Daten dieser Studie gezeigt, dass das Polnische in die gleiche Gruppe wie das Russische fällt, indem es auch die Verlaufsperspektive verwendet (vgl. die Daten aus Fedus 2006). Die gleiche Tendenz weist auch eine andere westslawische Sprache, das Slowakische, auf. Wie im Polnischen lässt sich auch in den slowakischen Daten eine Präferenz für die Enkodierung des Verlaufs feststellen. Diese Ergebnisse lassen vermuten, dass die Trennungslinie zwischen ost- und westslawischen Sprachen hinsichtlich des Grammatikalisierungsgrades von Aspekt möglicherweise verfeinert werden muss (siehe Kap. 4). 
Befunde zeigen, dass die perfektive Präsensform mit der Hier-und-jetzt-Bedeutung durchschnittlich in 12\% der Fälle gewählt wurde, und das unabhängig von der Verbklasse und der regionalen Zugehörigkeit der Probanden. Eine parallele Studie zum Russischen ergab, dass muttersprachliche Probanden die perfektive Präsensform in Verbindung mit der Hier-und-jetzt-Bedeutung nie verwenden (Kap. 3).

\subsection{Die Studie}

Es soll untersucht werden, wie zielorientierte Bewegungsereignisse im Deutschen, Tschechischen und Russischen unter einer verkürzten Zeitbedingung verbalisiert werden. Es wird angenommen, dass die Planung von Redeinhalten unter Zeitdruck anders verläuft als unter Bedingungen, in denen dem Probanden ausreichend oder sogar unbegrenzt viel Zeit für die Planung der Redeinhalte zur Verfügung steht. ${ }^{98}$ In den vorherigen Studien (Kap. 4-5) haben Probanden Videoclips gesehen, zwischen welchen 5- bzw. 8-sekundige Pausen bestanden. In der vorliegenden Studie wurde der Abstand zwischen den Videoclips auf eine 3-sekundige Pause reduziert.

Die Erwartung war, dass unter einer zeitlich sehr eingeschränkten Bedingung nur die für die jeweilige Sprache notwendigen Informationen von Probanden ausgewählt und verbalisiert werden. Dabei stellt sich die Frage, auf welche Informationen bzw. Szenenkomponenten in der jeweiligen Sprache verzichtet werden kann, ohne dass es zu einer unüblichen, sprachlich auffälligen oder sogar ungrammatischen Aussage kommt. Des Weiteren sollte auch untersucht werden, ob und zu welchen anderen sprachlichen Strukturen, wie z.B. Substantivierungen oder Adjektivierungen, Probanden im Zeitdruck-Paradigma greifen würden. Konkret stellen sich die folgenden Forschungsfragen:

1. Wird das Tschechische und das Deutsche die Endpunktmarkierung, und somit die holistische Perspektive, auch unter Zeitdruck beibehalten? Es gibt andere plausible Alternativen, die Muttersprachler beider Sprachen wählen können. Zum Beispiel könnten sie, anstatt den Endpunkt in einem zielorientierten Bewegungsereignis $\mathrm{zu}$ inferieren und $\mathrm{zu}$ markieren, die Angabe der Trajektorie (path) (z.B. Zwei Frauen laufen eine Straße entlang/an einem Haus vorbei), des Ortes (location) (z.B. Zwei Frauen laufen auf der Straße/im Park),

98 Eine Aufgabe, für deren Bewältigung die Probanden unbegrenzt Zeit haben, wird als Offline bezeichnet. Ein Beispiel dafür wäre eine schriftliche Nacherzählung eines Kurzfilmes nach dessen Ablauf (vgl. Schmiedtová/Sahonenko 2012). 
oder der Art und Weise (manner) (z.B. Zwei Frauen laufen schnell/gemütlich / zusammen) wählen. Dabei ist zu betonen, dass die Wahl von alternativen Angaben zwingend dazu führt, dass die Situation nicht unter holistischer Perspektive wiedergegeben werden kann.

2. Es stellt sich außerdem die Frage, ob die Zeitdruck-Bedingung im Tschechischen und Deutschen die Verwendung von imperfektiven Verbformen in einer 'nackten', d.h. nur Subjekt und Verb, aber keine weiteren Argumente oder Adjunkte umfassenden Verbalphrase, möglich macht. In Schmiedtová (2013a: 152-155) wurde gezeigt, dass solche nackten Verbalphrasen in sprachlichen Enkodierungen ohne Zeitdruck weder im Tschechischen noch im Deutschen vorkommen, dafür aber im Russischen durchaus gebraucht werden (z.B. rus. Mašina jed'et Ø - dt. Ein Auto fährt Ø).

3. Drittens stellt sich die Frage, ob und inwieweit die Endpunktmarkierung als Folge des Zeitdrucks im Russischen zu erklären ist. Ohne Zeitdruck werden in zielorientierten Bewegungsereignissen Endpunkte durchschnittlich in 46\% (siehe Kap. 5) bzw. in 63\% (siehe Kap. 8) ${ }^{99}$ aller Äußerungen von russischen Muttersprachlern markiert. Das heißt, dass immerhin etwa die Hälfte der getesteten Probanden eine Endpunktmarkierung in kritischen Stimuli vornahm.

Es könnte aber sein, dass die russischen Probanden, wenn sie unter Zeitdruck stehen, auf die Endpunktmarkierung in kritischen Stimuli verzichten. Wenn sich also der Zeitdruck so auswirkt, dass es zu einer Reduzierung der Endpunktmarkierungen im Russischen kommt, stellt sich die Frage, welche Information stattdessen verbalisiert wird. Könnten wir annehmen, dass unter Zeitdruck im Russischen die Verwendung von nackten Verbalphrasen zunimmt?

Angesichts der Tatsache, dass sehr fortgeschrittene russische Lerner des Deutschen die nackten Verbalphrasen in der Zielsprache auch ohne Zeitdruck relativ häufig verwenden und dieser Befund als ein Transfereffekt aus der Muttersprache gedeutet wird (Schmiedtová 2013a: 160), erscheint diese Annahme plausibel.

Zusammenfassend kann gesagt werden, dass die vorliegende Studie zum Ziel hat, mit Hilfe des Zeitdruck-Paradigmas die Beständigkeit der Endpunkmarkierung unter Verwendung der holistischen Perspektive in allen drei Sprachen zu

99 Der Unterschied in den Ergebnissen der Endpunktmarkierung zwischen den zwei Studien ist vermutlich mit den etwas unterschiedlich gestalteten Stimuli zu erklären. Festzuhalten ist, dass in beiden Studien russische Probanden statistisch signifikant weniger Endpunkte erwähnt haben als deutsche und tschechische Probanden. 
testen. Es wird davon ausgegangen, dass der Zeitdruck die Versprachlichung von Bewegungsereignissen ohne sichtbaren Endpunkt in allen drei zu untersuchenden Sprachen beeinflusst, dieser Einfluss sich jedoch unterschiedlich auswirkt: Im Tschechischen und Deutschen wird eine relative Stabilität der holistischen Perspektivierung erwartet, während für das Russische eine Reduzierung der Endpunktmarkierung und eine gleichzeitige Zunahme des Gebrauchs von nackten Verbalphrasen anzunehmen ist. Offen bleibt, welche anderen sprachlichen Strukturen, wie z.B. Nominalisierungen, unter Zeitdruck in allen drei Sprachen möglicherweise gebraucht werden, sowie die Frage nach der Verwendung der nackten Verbalphrasen im Tschechischen und Deutschen.

Die methodische Vorgehensweise für die Erforschung dieser Fragestellungen wird im folgenden Abschnitt erläutert.

\subsection{Experiment}

\subsubsection{Probanden}

Für die Untersuchung wurden insgesamt 83 Probanden aufgenommen, die sich in drei Gruppen mit 22 deutschen, 41 tschechischen und 20 russischen Sprechern gliedern. Alle Probanden füllten einen Fragebogen aus, in dem biografische Daten und soziale Variablen ermittelt wurden. Dabei sollten die sozialen Variablen Alter und Bildung bei den Versuchspersonen möglichst konstant gehalten werden. Aus diesem Grund wurden für das Experiment Erwachsene im Alter von 19 bis 36 Jahren, meist Studierende (Durchschnittsalter: Deutsch 24,8 Jahre, im Bereich von 20 bis 30 Jahre; Tschechisch 24,6 Jahre, im Bereich von 19 bis 36 Jahre; Russisch 26,1 Jahre, im Bereich von 22 bis 33 Jahre) herangezogen, deren Erstspracherwerb bereits abgeschlossen war. Die tschechischen und deutschen Daten wurden im jeweiligen Land erhoben, d.h. in Prag (Tschechien) und in Heidelberg (Deutschland). Die Daten der russischen Muttersprachler wurden aus logistischen Gründen in Heidelberg aufgenommen. Diese Probanden hatten Russisch als Muttersprache, kamen im Erwachsenenalter nach Deutschland (d.h. sie waren älter als 16 Jahre) und verfügten über sehr gute oder gute Deutschkenntnisse. ${ }^{100}$ Um weitestgehend gewährleisten $\mathrm{zu}$ können, dass sich die russischen Probanden während der Aufnahme im russischen, und nicht im deutschen Sprachmodus (Grosjean 1995, 2001) befanden, wurde das gesamte Experiment,

100 Die Tatsache, dass die russischen Probanden auch Deutsch konnten, war aufgrund des Aufnahmeortes nicht zu vermeiden. 
einschließlich eines warming-up-Gespräches und eines kurzen Nachgespräches, von einer russischen Muttersprachlerin auf Russisch durchgeführt. Die Geschlechterverteilung in allen Gruppen war mit etwa doppelt so vielen weiblichen als männlichen Probanden in jeder der drei Gruppen nicht gleichmäßig.

\subsubsection{Experimentablauf}

Die Untersuchung bestand aus einer sprachlichen Aufgabe, in der Muttersprachler kurze Videoclips nacherzählen sollten. Das Stimulusmaterial umfasste insgesamt 37 Stimuli: Zum einen waren es kritische Stimuli $(n=10)$, bei denen Unterschiede zwischen den getesteten Sprachen erwartet wurden; zum anderen die so genannten Fillers $(n=27)$, die zur Ablenkung dienten. ${ }^{101}$ Die kritischen Stimuli waren zielorientierte Bewegungsereignisse, in denen eine Bewegung belebter Entitäten (Menschen oder Tiere) von Punkt A zu Punkt B als nicht erreicht gezeigt wurde [-END].

Die Stimuli wurden als Videoclips in einem Durchgang dargeboten. Die Probanden wurden aufgefordert, die Frage dt. Was passiert gerade?, tsch. Co se děje? bzw. rus. Čto sejčas proischodit? zu beantworten, sobald sie die Antwort geben konnten. Ein Experimentdurchgang dauerte ungefähr 15 Minuten und begann mit einer kurzen Trainingsphase. Für die Datenerhebungen wurden die Stimuli randomisiert, sodass sich für jeden Probanden eine individuelle Stimulusabfolge ergab. Das akustische Signal wurde zunächst digital aufgenommen und transkribiert. Anschließend wurden die transkribierten Daten kodiert und analysiert.

\subsubsection{Kodierung der Daten}

In allen drei Datensets wurde die Markierung der Endpunkte, der Art und Weise (z.B. schnell, gemütlich), des Ortes (z.B. auf der Straße, im Park), der Trajektorie (z.B. entlang, vorbei) sowie der Ausgangspunkte kodiert. Darüber hinaus wurde auch das Vorkommnis der nackten Verbalphrasen erfasst. Es folgen Beispiele für die Kodierungskategorien Endpunkt (END), Trajektorie und Ausgangspunkt aus allen drei Sprachen.

101 Beispiele für kritische Stimuli und Fillers sind dem Anhang D zu entnehmen. 


\section{Markierung der Endpunkte (END)}

(20) dt.

VP06_dt. ${ }^{102}$

(21) tsch.

VP09_tsch.

(22) rus.

VP02_rus.
Eine Frau geht zum Auto

Žena jde

ke svému autu

Frau.Sg.Nom geh.Präs.Impf.3.Sg

zu ihr.Dat.Poss.Refl Auto.Sg.Dat

'Eine Frau geht zu ihrem Auto'

Devushka idjoet

po napravleniju $k$ mashine

Mädchen.Sg.Nom geh.Präs.Impf.3.Sg

in Richtung.Akk zu Auto.Sg.Dat

'Ein Mädchen geht in Richtung eines Autos'

Als Endpunkt wurden alle Präpositionsphrasen gezählt, in denen der inferierbare Endpunkt erwähnt wurde, einschließlich Präpositionsphrasen mit den Präpositionen in Richtung, $z u$, in, nach, auf $X z u$.

\section{Markierung der Trajektorie (TRAJ)}

(23) dt.

VP15_dt.

(24) tsch.

VP14_tsch.

(25) rus.

VP17_rus.
Ein Auto fährt eine Straße entlang

Auto jede vesnicí

Auto.Sg.Nom fahr.Präs.Impf.3.Sg Dorf.Sg.Ins

'Ein Auto fährt durch ein Dorf'

Mashina edet

po sagorodnoj doroge

Auto.Sg.Nom fahr.Präs.Impf.3.Sg

auf Landstraße.Sg.Dat

'Ein Auto fährt eine Landstraße entlang'

Als Markierung der Trajektorie wurden im Tschechischen und Russischen auch die mit dem Präfix pro- ('durch') markierten Verben kodiert (z.B. tsch. Auto pro-

102 Die Zahl bezieht sich auf die Nummerierung der Probanden in der jeweiligen Probandengruppe. 
jelo - 'Ein Auto fuhr vorbei'). Solche Fälle kamen aber nur selten vor, da präfigierte Verben meistens in Kombination mit anderen Angaben verwendet wurden (z.B. tsch. Auto pro-jelo (Weg=Trajektorie) do vesnice (Endpunkt) - 'Ein Auto fuhr vorbei, in ein Dorf'). Als Trajektorie wurden alle Präpositionsphrasen mit den Präpositionen über, durch, vorbei etc. gezählt.

\section{Markierung der Ausgangspunkte (AUSG)}

(26) dt. Eine Frau kommt mit einem Einkaufswagen VP16_dt. aus einem Einkaufsladen

(27) tsch. Holka jede z obchodu s vozíkem

VP42_tsch. Mädel.Sg.Nom fahr.Präs.Impf.3.Sg aus Geschäft.Sg.Gen mit Einkaufswagen.Sg.Ins

'Ein Mädel fährt aus einem/dem Geschäft mit einem Einkaufswagen'

$\begin{array}{ll}\text { rus. } & \begin{array}{l}\text { Devushka idjoet } \\ \text { sakupochnoj kolaskoj is magasina }\end{array} \\ \text { VP12_rus. } & \text { Mädchen.Sg.Nom geh.Präs.Impf.3.Sg mit } \\ & \text { Einkaufswagen.Sg.Ins aus Geschäft.Sg.Gen } \\ & \text { 'Ein Mädchen geht mit einem Einkaufwagen aus einem/ } \\ \text { dem Geschäft' }\end{array}$

\section{Kombinationen}

In manchen Fällen wurden mehrere (zwei oder drei) Markierungen im gleichen Satz kombiniert. Wenn es zu Kombination mehrerer Markierungen kam, wurde nur eine Markierung gezählt. Dabei galt die folgende Hierarchie: ${ }^{103}$ Endpunkt > Trajektorie > Ort > Art und Weise. Eine in der Äußerung vorhandene Endpunktmarkierung überschrieb bei der Kodierung der Daten somit die Angabe anderer Informationen. Die Frage, in welchen Sprachen welche Angaben kombiniert wurden, wird in Abschnitt 6.5 erörtert.

103 Da in dieser Studie die Endpunktmarkierung untersucht wurde - die verwendeten Stimuli wurden so konzipiert, um die Endpunktmarkierung gezielt zu elizitieren -, wurden die Endpunkte in der Kodierungshierarchie ganz oben platziert, gefolgt von der Trajektorie, die als eine dynamische Qualität im Vergleich zu dem Ort (statische Qualität) als für Bewegungsereignisse relevanter betrachtet wird. 


\section{Keine Markierung (KEINE)}

Die Verwendung nackter Verbalphrasen wurde unter der Kategorie KEINE erfasst. Zum Beispiel: rus. Poesd/Tramvaj edet Ø - dt. 'Ein Zug/Eine Straßenbahn fährt' Ø‘; rus. Dve monashki idut Ø - dt. 'Zwei Nonnen laufen/gehen Ø’; tsch. Pes běži Ø - dt. 'Ein Hund läuft $\emptyset$ ’.

\section{Andere Formen (ANDERE)}

Es wurden auch Adjektivierungen und Nominalisierungen in den Daten gefunden. Diese wurden zum einen separat ausgewertet (siehe Kap. 5), zum anderen wurden diese Formen (wenn möglich) in das Kodierungssystem integriert. So wurde z.B. die Verwendung einer Adjektivierung mit Endpunktangabe bzw. Trajektorieangabe als Endpunkt- bzw. Trajektoriemarkierung gewertet: tsch. $z a$-jiždějící (Endpunkt) auto - 'ein einfahrendes Auto'; tsch. přes dvưr (Trajektorie) běžící pes 'über den Hof laufender Hund'.

\section{Nicht kodierte Äußerungen}

Antworten, in denen nur Nomen ohne verbale Elemente produziert wurden (z.B. Hund), oder Antworten, die die gestellte Frage Was passiert? nicht beantwortet haben (z.B. Interpretationen), sowie wortlose Reaktionen wurden unter die Kategorie Andere gefasst. Sie werden in den Abbildungen in Abschnitt 6.5 separat aufgeführt.

\subsection{Ergebnisse}

In diesem Abschnitt werden die Ergebnisse der vorliegenden Studien vorgestellt. Der Hauptfokus liegt dabei auf der quantitativen Analyse; am Ende dieses Kapitels wird eine qualitative Analyse der Verwendung von Adjektivierungen und Nominalisierungen im Deutschen und Tschechischen präsentiert.

Zuerst wird die Verteilung der analysierten Kategorien in jeder Sprache einzeln analysiert, anschließend der zwischensprachliche Vergleich. Die Datenpräsentation folgt der Reihenfolge Deutsch $>$ Tschechisch $>$ Russisch.

Aus Abbildung 20 lässt sich entnehmen, dass im Deutschen hauptsächlich drei Kategorien eine Rolle bei der Enkodierung von zielorientierten Bewegungsereignissen unter Zeitdruck spielen: Markierung des Endpunktes kommt in beinahe der Hälfte aller Antworten vor, die Angabe der Trajektorie in einem Viertel der produzierten Äußerungen. Der Ort des Geschehens wird etwa in einem Sechstel der Daten angegeben. Die Markierung des Ausgangspunktes ist eher marginal; die Art und Weise wird von deutschen Muttersprachlern gar nicht zum Ausdruck gebracht. Im Deutschen besteht, auch unter Zeitdruck, eine Präferenz für End- 
punktmarkierung: $\left(\chi^{2}(6)=132,1, p<.05\right) .{ }^{104}$ Darüber hinaus gibt es eine kleine Anzahl von Äußerungen (5\%) mit nackten Verbalphrasen, deren Verwendung weiter unten diskutiert wird.

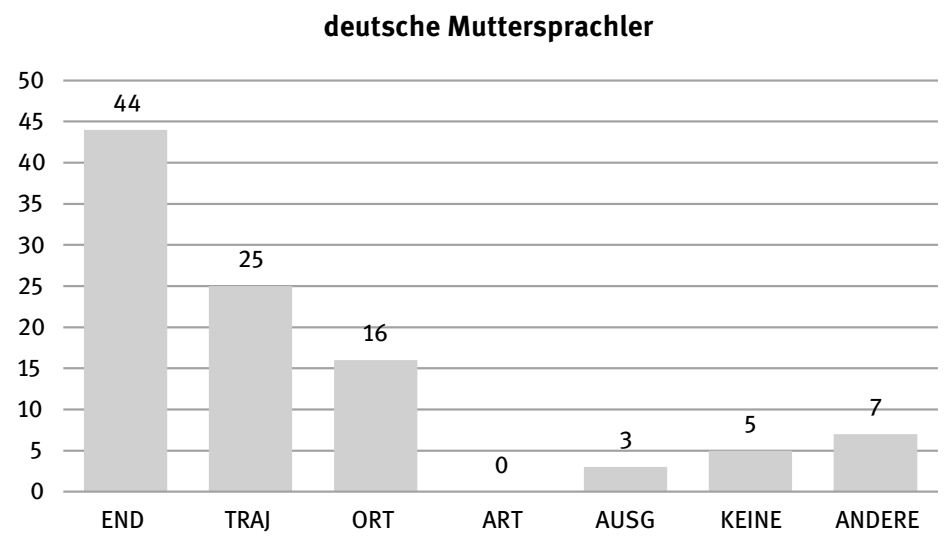

Abb. 20: Enkodierung zielorientierter Bewegungsereignisse - deutsche Muttersprachler

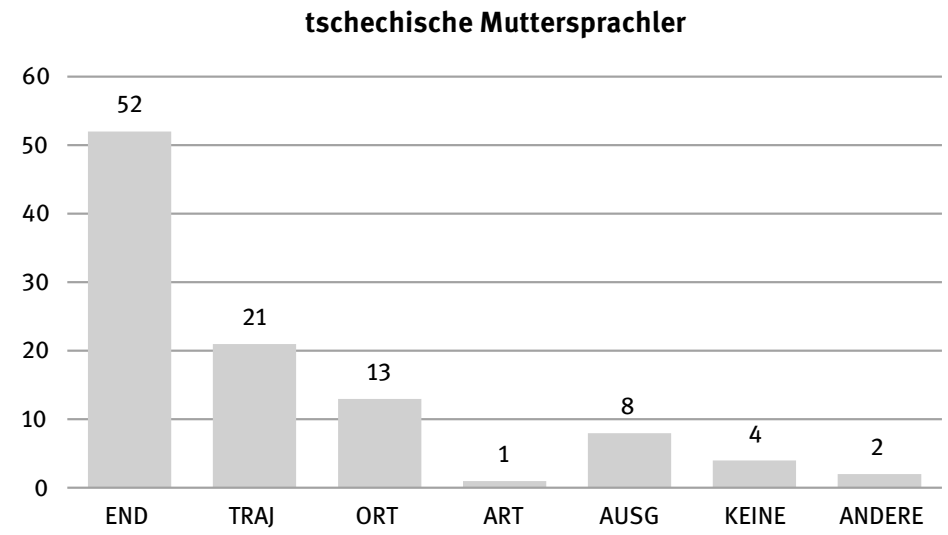

Abb. 21: Enkodierung zielorientierter Bewegungsereignisse - tschechische Muttersprachler

104 Um bestimmen zu können, welche Kategorien am stärksten zur Ablehnung der Nullhypothese beigetragen haben, wurden zusätzlich für die statistisch relevanten Kategorien, END und TRAJ, die so genannten absoluten Residuen (R) berechnet und verglichen. (Wenn $\mathrm{R}>2$, ist die Kategorie relevant.) Für [END] war $R=11,5$; für TRAJ $R=4,0$. 
Aus den tschechischen Daten (Abb. 21) geht hervor, dass so wie in den deutschen Daten die Markierung der Endpunkte, der Trajektorie und des Ortes die am häufigsten produzierten Angaben darstellen. Die Anzahl der Äußerungen mit der Angabe der Art und Weise sowie der Ausgangspunktmarkierung sind seltener (1\% bzw. 8\%). In 4\% aller Äußerungen kommen nackte Verbalphrasen vor. Die Kategorie Andere umfasst lediglich 2\% aller Äußerungen. Es lässt sich festhalten, dass die Verteilung der analysierten Kategorien in den tschechischen und den deutschen Daten starke Ähnlichkeiten aufweist. Dies wird auch durch die für die tschechischen Daten festgestellte Präferenz für die Markierung der Endpunkte gestützt $\left(\chi^{2}(6)=401,6, p<.05\right) .{ }^{105}$

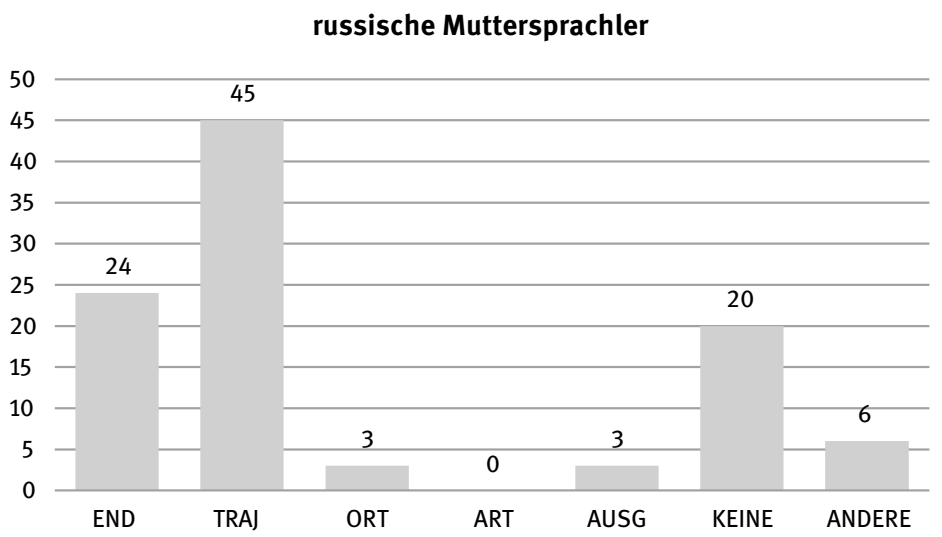

Abb. 22: Enkodierung zielorientierter Bewegungsereignisse - russische Muttersprachler

Im Vergleich zum Deutschen und Tschechischen ergibt sich für die russischen Daten ein vollkommen anderes Bild. Die Abbildung 22 zeigt eine anders gewichtete Verteilung in der Verwendung der Kategorien: Am häufigsten wird die Trajektorie zum Ausdruck gebracht. Endpunkte werden in etwa einem Viertel der produzierten Äußerungen markiert. Die Angabe des Ortes und des Ausgangspunktes spielen eine untergeordnete Rolle, die Art und Weise wurde nicht markiert. Ein markanter weiterer Unterschied zum Deutschen und Tschechischen besteht darin, dass 'nackte' Verbalphrasen relativ häufig verwendet werden (20\%). Das

105 So wie für die deutschen Daten wurden auch hier für die statistisch relevanten Kategorien, END und TRAJ, die absoluten Residuen (R) kalkuliert: für END war $R=20,2$; für TRAJ $R=3$,9. 
Russische zeigt insgesamt jedoch eine Präferenz für die Markierung der Trajektorie: $\left(\chi^{2}(6)=127,6, p<.05\right){ }^{106}$

Der Vergleich der drei Sprachen hinsichtlich der Endpunktmarkierung hat keinen statistisch relevanten Unterschied zwischen dem Deutschen und dem Tschechischen $\left(\chi^{2}(1)=3,7 ; n s\right)$ ergeben. Im Gegensatz dazu waren die Vergleiche zwischen Deutsch und Russisch einerseits und Tschechisch und Russisch andererseits durchaus signifikant: dt. vs. rus. $\left(\chi^{2}(1)=17,9, p<.05\right)$; tsch. vs. rus. $\left(\chi^{2}(1)=\right.$ 42,2, $p<.05)$. Für die Markierung der Trajektorie konnte das umgekehrte Muster festgestellt werden. Im Russischen kommt die Angabe der Trajektorie viel häufiger vor als in den anderen zwei Sprachen: rus. vs. dt. $\left(\chi^{2}(1)=17,7, p<.05\right)$; rus. vs. tsch. $\left(\chi^{2}(1)=34,6, p<.05\right)$. Zwischen dem Deutschen und dem Tschechischen wiederum gibt es hinsichtlich der Markierung der Trajektorie keinen statistisch relevanten Unterschied: dt. vs. tsch. $\left(\chi^{2}(1)=1,02, n s\right)$.

Im Folgenden wird die Verwendung der nackten Verbalphrasen in den drei Sprachen näher betrachtet (Abb. 23).

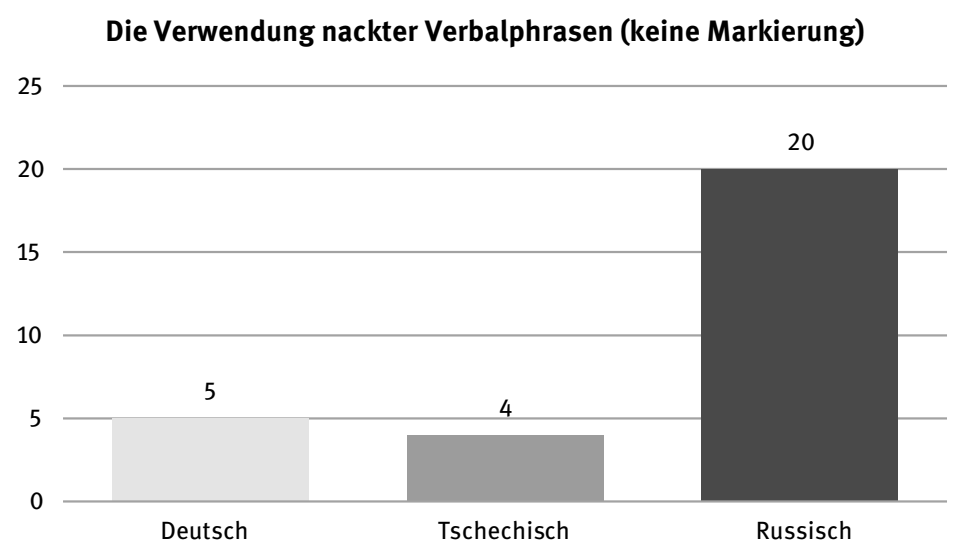

Abb. 23: Verwendung nackter Verbalphasen - deutsche, tschechische, russische Muttersprachler

106 Da TRAJ in den russischen Daten die einzige statistisch relevante Kategorie war, mussten keine absoluten Residuen gerechnet werden. 
Obwohl nackte Verbalphrasen in den Daten aller drei Sprachen gefunden werden konnten, variiert deren Häufigkeit sehr stark. Die statistischen Analysen ergeben, dass das Russische sich von den anderen zwei Sprachen signifikant unterscheidet: rus. vs. dt. $\left(\chi^{2}(1)=250,4, p<.05\right)$; rus. vs. tsch. $\left(\chi^{2}(1)=382, p<.05\right)$. Demgegenüber ergab der Vergleich Deutsch und Tschechisch keinen signifikanten Unterschied: $\left(\chi^{2}(1)=0,15, n s\right)$.

Das Vorkommen anderer Äußerungen, die in der Kategorie ANDERE erfasst wurden, ist in allen drei Sprachen vergleichbar niedrig (dt. 7\%; tsch. 2\%; rus. 6\%), was als ein Hinweis auf die Tauglichkeit der verwendeten Stimuli sowie auf die Angemessenheit der Aufgabe interpretiert wird.

Zum Gebrauch mehrerer Markierungen in Kombination lässt sich festhalten, dass diese ausschließlich in den deutschen und den tschechischen Daten vorkamen. Sprecher beider Sprachen verwenden kombinierte Markierungen trotz der Zeitdruck-Bedingung. Aus den Daten geht hervor, dass deutsche und tschechische Sprecher vergleichbar of $\mathrm{t}^{107}$ verschiedene Markierungen kombinieren: dt. $19 \%(n=36)$ aller markierten Äußerungen $(n=194)$; tsch. $16 \%(n=62)$ aller markierten Äußerungen $(n=384)$.

\section{deutsche Muttersprachler}

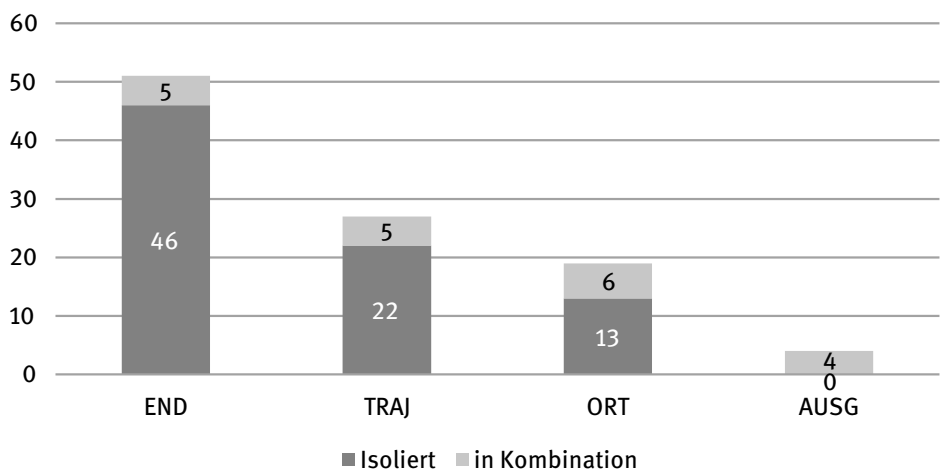

Abb. 24: Verwendung mehrerer Markierungen in Kombination - deutsche Muttersprachler

107 Der Unterschied zwischen Deutsch und Tschechisch ist statistisch nicht relevant: $\left(\chi^{2}(1)=\right.$ $0,5, n s)$. 


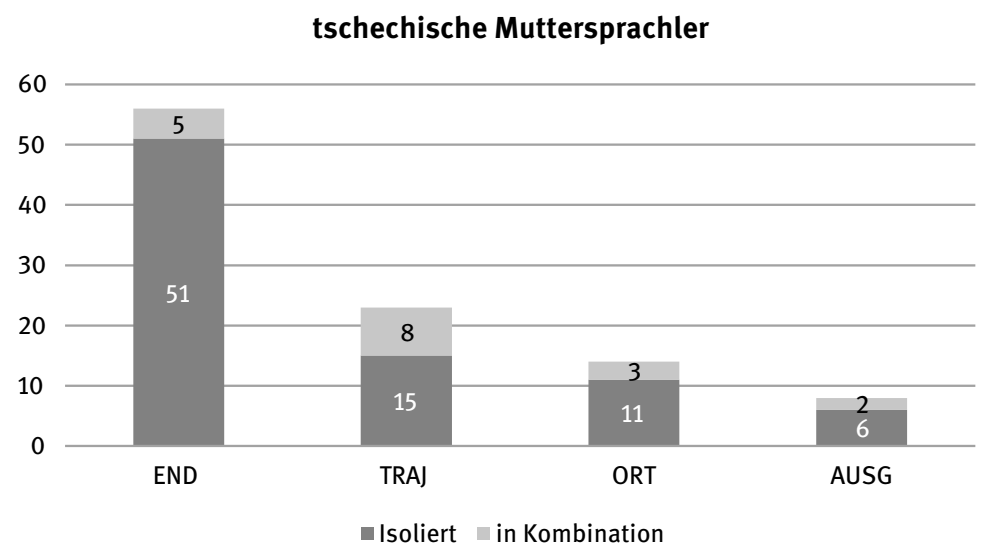

Abb. 25: Verwendung mehrerer Markierungen in Kombination - tschechische Muttersprachler

Bei näherer Betrachtung der Abbildungen 24 und 25 wird ersichtlich, dass in beiden Sprachen am häufigsten Angaben zu Endpunkten, der Trajektorie sowie des Ortes kombiniert werden. Zur Kombination mit der Markierung von Ausgangspunkten soll zweierlei angemerkt werden: 1) Im Deutschen werden diese nur in Kombination und nicht isoliert gebraucht; 2) in beiden Sprachen kommt diese Kombination bei der Beschreibung einer bestimmten Szene vor: Eine Frau befindet sich auf dem Parkplatz eines Supermarktes und läuft mit ihrem Einkaufswagen zu ihrem Auto. Was die Kombination von drei Markierungen anbetrifft, kam diese nur zweimal, und zwar in den tschechischen Daten vor.

(a) Kombination von zwei Markierungen

(29) dt. Eine Frau geht über einen Parkplatz (Trajektorie) zu einem Auto (Endpunkt)

(30) tsch. Pes pro-běhl (Trajektorie) do dveří (Endpunkt) Hund.Sg.Nom lauf.Prt.Perf.3.Sg in Tür.Sg.Akk 'Ein Hund lief vorbei, in eine Tür rein'

(b) Kombination von drei Markierungen

(31) tsch. Auto od-jižzí (Ausgangspunkt)na venkově (Ort) směrem ke vsi (Endpunkt) Auto.Nom fahr.Präs.Impf.3.Sg auf Land.Sg.Dat in Richtung zu Dorf.Sg.Lok 'Ein Auto fährt weg, auf dem Lande, in Richtung Dorf' 
Abschließend soll der Gebrauch anderer Formen betrachtet werden: Dabei handelt es sich um Adjektivierungen und Nominalisierungen bzw. Nominalformen. Diese Formen kamen nur selten vor und wurden nur in den deutschen und den tschechischen Daten gefunden (dt. $n=10$; tsch. $n=12$ ). Zusätzlich wurden diese Formen von lediglich sieben deutschen und vier tschechischen Probanden produziert. Es fällt auf, dass solche Reduzierungen überwiegend in Verbindung mit unbelebten Entitäten gebraucht werden. Hier einige Beispiele aus den erhobenen Daten:

Deutsche Daten

- das Parken eines Autos

- ein anfahrender Zug am Bahnhof

- fahrendes Auto an einer Landstraße in der dreißiger Zone

- ein rollender Fahrradreifen

- eine Frau beim Einkaufen

Tschechische Daten

- leticí kolo potrávě

fliegend.Nom Rad.Nom auf Gras.Sg.Lok

'ein durch das Gras fliegendes Rad'

- dvě kamarádky procházejícíse poparku

zwei.Fem Freundin.Pl durchlaufend.Nom.Refl durch Park.Sg.Lok

'zwei durch den Park spazierende Freundinnen'

- běžicí pes do zelených vrat

laufend.Sg.Nom Hund.Sg.Nom in grün.Gen Tor.Sg.Gen

'ein in grünes Tor rennender Hund'

- vlak jedoucí po kolejích

Zug.Sg.Nom fahrend.Nom auf Gleis.Pl.Lok

'ein auf Gleisen fahrender Zug'

Obwohl diese Formen nicht häufig sind, scheint die Zeitdruck-Bedingung charakteristisch für ihren Gebrauch zu sein; unter schwächeren Zeitbedingungen (5oder 8-sekundige Pausen) kommen sie nicht vor. Es ist darüber hinaus bemerkenswert, dass sie ausschließlich von deutschen und tschechischen, jedoch nicht von russischen Probanden gebraucht werden.

Zusammenfassend ist festzuhalten, dass deutsche und tschechische Probanden auch unter Zeitdruck die holistische Perspektive verwenden. Im Gegensatz zu russischen Probanden präferieren deutsche und tschechische Sprecher die Markierung von Endpunkten. Darüber hinaus wurden weitere Ähnlichkeiten zwischen dem Deutschen und Tschechischen festgestellt: Beide Sprachen verwen- 
den auch mehrere Markierungen in Kombination sowie andere Formen (z.B. Adjektivierungen) zur Beschreibung der dargebotenen Stimuli. Dies trifft für das Russische nicht zu. Die russischen Produktionsdaten zeichnen sich durch eine Präferenz für die Markierung der Trajektorie sowie durch einen sehr hohen Gebrauch von nackten Verbalphrasen aus.

\subsection{Fazit und Diskussion}

Die Studie hat deutlich gemacht, dass auch im Zeitdruck-Paradigma Sprecher unterschiedlicher Sprachen bei der Beschreibung desselben Ereignisses systematisch auf sprachspezifische Muster zurückgreifen. Insbesondere konnte gezeigt werden, dass deutsche und tschechische Muttersprachler bei der Verbalisierung zielorientierter Bewegung bevorzugt Endpunkte zum Ausdruck bringen und somit diese Ereignisse unter holistischer Perspektive wiedergeben. Diese Befunde bestätigen die Ergebnisse früherer Studien (Schmiedtová 2010; 2011a, 2011b; Schmiedtová/von Stutterheim/Carroll 2011; Kap. 5 und 8), in denen wiederholt demonstriert wurde, dass das Tschechische nicht etwa mit dem Russischen (einer Verlauf-Sprache), sondern mit dem Deutschen (einer Endpunkt-Sprache) ein Cluster bildet. Im Hinblick auf die Ergebnisse anderer in diesem Kapitel zitierten Studien zu Blickverhalten und Gedächtnisleistung ist auch für die vorliegende Studie anzunehmen, dass dieses Muster nicht nur für die sprachliche Enkodierung, sondern auch für die zugrundeliegende Konzeptualisierung gilt.

Die Beobachtung, dass das tschechische nicht mit dem russischen Muster einhergeht, lässt sich für Bewegungsereignisse nicht anhand der verwendeten Verben und der Aspektmarkierung untersuchen, da beide Sprachen für die Versprachlichung dieser Ereignisse überwiegend die gleichen Verben mit imperfektiver Bedeutung verwenden. Die unterschiedlichen zugrundeliegenden Präferenzen für die Enkodierung der Bewegungsereignisse schlagen sich jedoch in der Informationsauswahl und der gewählten Argumentstruktur nieder. ${ }^{108}$ Dies wird besonders unter der Zeitdruck-Bedingung deutlich, die bei tschechischen und russischen Sprechern zu unterschiedlichen Effekten führt.

108 Es soll betont werden, dass die gewählte Konzeptualisierung mit der in der jeweiligen Sprache zulässigen Argumentstruktur stark korreliert. Die Frage nach dem genauen Verhältnis zwischen den konzeptuellen Repräsentationen einerseits und den syntaktischen Eigenschaften andererseits muss aber wohl zu den berühmten Henne-Ei-Problemen gezählt werden und lässt sich nicht eindeutig beantworten. 
Das verbindende Glied zwischen dem Deutschen und dem Tschechischen liegt darin, dass beide Sprachen die Abgeschlossenheit von Ereignissen fokussieren: Das Merkmal Abgeschlossenheit wird in jeder auch nur potenziell abgeschlossenen Szene inferiert und die Situation systematisch unter holistischer Perspektive versprachlicht. Der Fokus auf Abgeschlossenheit schlägt sich für zielorientierte Bewegung in der Präferenz für die Markierung der Endpunkte nieder, welche in Form von Präpositionalphrasen und/oder mithilfe spezieller Präfixe ausgedrückt werden.

Es ist hier wichtig zu betonen, dass bei der Enkodierung anderer Arten von Ereignissen, z.B. Situationen mit einem Nachzustand, im Tschechischen das Merkmal Abgeschlossenheit mit Hilfe des perfektiven Aspekts (als perfektive Präsens- oder Vergangenheitsform) zum Ausdruck gebracht wird (siehe auch Kap. 2, 3 und 10). ${ }^{109}$ Neben dem gemeinsamen Fokus auf Abgeschlossenheit ähneln sich das Deutsche und das Tschechische auch in Bezug darauf, dass sie unter verkürzter Zeitbedingung verschiedene Markierungen kombinieren und somit eine höhere Informationsdichte als z.B. das Russische schaffen. Außerdem führt der Zeitdruck im Deutschen sowie im Tschechischen zur Verwendung von reduzierten sprachlichen Formen wie Adjektivierungen (mit Angabe der Endpunkte oder der Trajektorie), die im Russischen gar nicht gebraucht werden.

Abschließend werden die in Abschnitt 6.3 formulierten Forschungsfragen in verkürzter Form wieder aufgenommen:

1. Wird im Tschechischen und im Deutschen die Endpunktmarkierung, und somit die holistische Perspektive, auch unter Zeitdruck beibehalten?

Obwohl die Frage klar mit Ja zu beantworten ist, hat die Zeitdruck-Bedingung in beiden Sprachen einen klaren Effekt auf die Verbalisierung: (1) Die Gesamtzahl der Endpunktmarkierungen wird um etwa 25\% reduziert: So wurden z.B. in Schmiedtová/Sahonenko (2008) im Deutschen Endpunkte im Durchschnitt in $71 \%$, im Tschechischen $74 \%$ aller Äußerungen markiert. (2) Es scheint, dass im Deutschen und Tschechischen unter der Zeitdruck-Bedingung neben der Markierung der Endpunkte auch die der Trajektorie eine wichtige Rolle spielt. Andere Angaben dagegen, z.B. Ort oder Art und Weise, werden auch unter Zeitdruck kaum oder überhaupt nicht gebraucht.

109 Interessanterweise konnten sogar in der vorliegenden Studie drei Vorkommen von Vergangenheit Perfektiv und eines von Präsens Perfektiv in den tschechischen Daten gefunden werden (aber keines in den russischen). 
Der Zusammenhang zwischen der Informationswahl und der Perspektivierung in der Enkodierung von Bewegungsereignissen lässt sich anhand der folgenden Abbildung darstellen:

Fokus auf Verlauf

z.B. Russisch, Polnisch

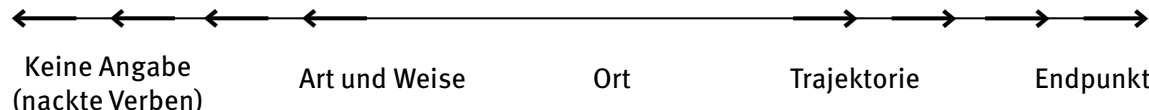

Abb. 26: Perspektivierung und Informationsselektion

Für die Verlaufssprachen lässt sich in Bezug auf die Aspektwahl und die Informationsselektion die Tendenz beobachten, dass unter normalen Zeitbedingungen der Gebrauch des imperfektiven Aspekts stark mit dem Ausdruck der Trajektorie und des Ortes korreliert (vgl. auch Carroll/von Stutterheim 2003 - für das Englische). Der Ausdruck der Trajektorie bleibt auch unter Zeitdruck erhalten und es steigt gleichzeitig der Gebrauch der nackten Verbalphrasen an. Das Tschechische, das eine Präferenz für die holistische Perspektive hat und über ein Aspektsystem verfügt, gibt ein anderes Bild ab: In Bewegungsereignissen, für die nur eine eingeschränkte Aspektwahl besteht, wird unter der normalen sowie der verkürzten Zeitbedingung 'das Holistische' vorwiegend mit Endpunktangabe wiedergegeben. Der Gebrauch der nackten Verbalphrasen spielt weder mit noch ohne Zeitdruck eine Rolle. Sobald tschechische Sprecher die Möglichkeit haben, aus mehreren aspektuellen Formen zu wählen (z.B. in der Enkodierung von Ereignissen mit Nachzustand), steigt der Gebrauch der perfektiven Formen sowohl in der Vergangenheit als auch im Präsens an (zur Verwendung der perfektiven Präsensform im Tschechischen siehe weiter unten und Kap. 3).

2. Ermöglicht die Zeitdruck-Bedingung im Tschechischen und Deutschen die Verwendung von imperfektiven Verbformen in einer nackten Verbalphrase?

Das Zeitdruck-Paradigma hat gezeigt, dass im Deutschen und Tschechischen die Verwendung der nackten Verbalphrasen nicht ausgeschlossen ist. Dies widerspricht der in Schmiedtová (2011a) und Schmiedtová/von Stutterheim/Carroll (2011) formulierten Annahme, die besagt, dass nackte Verbalphrasen (im gegebenen Kontext) weder im Deutschen noch Tschechischen grammatisch sind. Es ist dabei allerdings wichtig zu betonen, dass diese Verbalformen in den deutschen und tschechischen Daten nur selten, in den russischen Daten dagegen viel häufiger vorkommen. 
3. Inwieweit - wenn überhaupt - ist die Endpunktmarkierung im Russischen als Folge des Zeitdrucks zu bewerten?

Der Zeitdruck bewirkt im Russischen, dass die Markierung der Endpunkte stark zurückgeht. Dieser Befund lässt sich so interpretieren, dass die Endpunktmarkierung nicht zu den Kerninformationen gehört, die obligatorisch ausgedrückt werden. Dies bekräftigen auch die im Russischen häufig verwendeten nackten Verbalphrasen, die eine minimalisierte Argumentstruktur aufweisen, die aus zwei Komponenten besteht: Subjekt (Mensch oder Tier) und Verb (einfaches Imperfektiv).

Die Ergebnisse der in diesem Kapitel vorgestellten Studie werden auf den langandauernden Einfluss des Deutschen auf das Tschechische zurückgeführt. Die Daten deuten darauf hin, dass sich der Kontakt des Tschechischen zum Deutschen nicht auf den Bereich des Lexikons beschränkt hat, sondern auch im Bereich der Grammatik und auf der Ebene der zugrundeliegenden Konzepte tiefe Spuren hinterlassen hat. Neue Studien lassen vermuten (vgl. Dickey 2011; Schmiedtová/von Stutterheim/Carroll 2011, Kap. 3 und 4), dass auch komplexere sprachliche Bereiche im Tschechischen, z.B. allgemeine Diskurs- und Textprinzipien, durch den Kontakt mit dem Deutschen beeinflusst wurden.

Der genaue Verlauf und die Natur des Sprachkontakts zwischen dem Deutschen und dem Tschechischen sind natürlich nicht ganz einfach nachzuvollziehen oder darzustellen. Es scheint aber, dass sich der Kontakt zwischen dem Deutschen und dem Tschechischen anders gestaltete und auf jeden Fall zu unterschiedlichen Auswirkungen führte als beispielsweise der Kontakt des Deutschen mit dem Polnischen.

Die Sprachproduktionsdaten, die unter vergleichbaren Bedingungen und gleichem experimentellem Paradigma erhoben wurden, zeigen, dass im Polnischen, aber auch im Slowakischen, eine andere Perspektivierung für die Enkodierung von Bewegungsereignissen und Ereignissen mit Nachzustand eingesetzt wird, als dies im Tschechischen der Fall ist (siehe Kap. 4). Auch deuten tschechische Daten zum Gebrauch der perfektiven Präsensform auf eine Verschiebung der temporalen Interpretation hin: Weg von der perfektiven Zukunftsbedeutung hin zur de-perfektiven Präsensbedeutung (siehe Kap. 3). Diese Verwendung ähnelt der Verwendung der Partikelverben im Deutschen (z.B. Ich esse jetzt den Apfel auf). Im Gegensatz dazu weisen russische Daten keine Verschiebung der temporalen Bedeutung der perfektiven Präsensform auf. Es scheint also plausibel anzunehmen, dass die Grammatikalisierung des Aspekts im Tschechischen weniger fortgeschritten ist als im Russischen.

Weiterhin zeigen die Daten, dass polnische Muttersprachler im Gegensatz zu Muttersprachlern des Tschechischen für die Enkodierung von unterschiedlichen Ereignistypen perfektive Präsensformen weder in Vergangenheit noch Präsens 
verwenden und dass die im Tschechischen vorwiegend verwendete Perspektivierung anders ist als die im Polnischen. Was macht also den deutsch-tschechischen Kontakt so besonders? Im Rahmen der vorliegenden Studie, die den Einfluss synchron und anhand von empirischen Daten untersucht, lässt sich diese Frage gewiss nicht umfassend beantworten. Mit Sicherheit kann man jedoch festhalten, dass der über Jahrhunderte andauernde deutsch-tschechische Kontakt in vielen tschechischen Gebieten zu funktioneller Bilingualität führte (vgl. Fürst 2003; Trost 1965). Die gelebte Zweisprachigkeit, der enge kulturelle Kontakt zwischen den beiden Sprachen sowie zahlreiche weitere psychosoziale und soziolinguistische Faktoren waren maßgeblich für die Formung und Entwicklung des tschechischen Sprachsystems, deren Auswirkungen im heutigen Tschechisch zu beobachten sind.

\subsection{Zusammenfassung}

Dieses Kapitel umfasst Sprachproduktionsdaten von deutschen, tschechischen sowie russischen Muttersprachlern. Die Sprecher wurden mit der Aufgabe konfrontiert, unter verkürzter Zeitdruck-Bedingung kurze Videoclips mit Bewegungsereignissen zu versprachlichen. Die diesem experimentellen Paradigma zugrundeliegende Idee ist, dass Sprecher unter Zeitdruck nur die notwendigen Informationen zum Ausdruck bringen. Die Ergebnisse zeigen, dass das Tschechische und das Deutsche anders als das Russische die holistische Perspektive für die Versprachlichung der Bewegungsereignisse verwenden. In einem zweiten Schritt werden die Ergebnisse der vorliegenden Studie sowie ihre Hauptthese über den Einfluss des Deutschen auf das Tschechische vor dem Hintergrund von Sprachproduktionsdaten aus dem Polnischen diskutiert, sowie auf den Grammatikalisierungsgrad des Aspekts in verschiedenen slawischen Sprachen bezogen. 